\title{
Measurement of the turbulent diffusivity in the near field of variable density jets using conditional velocimetry
}

\author{
D. Stepowski, J.C. Sautet
}

Abstract Pulsed laser Mie scattering and laser Doppler velocimetry (LDV), both conditioned on the origin of the seed particles, have been successively performed in turbulent jets with variable density. In the early stages of the jet developments, significant differences are measured between the ensemble average LDV data obtained by jet seeding and those obtained by seeding the ambient air. Careful analysis of the marker statistics shows that this difference is a quantitative measure of the turbulent mixing. The good agreement with gradient-diffusion modelling suggests the validity of a general diffusion equation where the velocities involved are expressed in terms of ensemble conditional Favre averages. This operator accounts for all events (including intermittent ones) and for variations in the density of the marked fluid whose velocity is still specified by the binary origin of the marker.

\section{List of symbols}

$D_{\mathrm{L}} \quad$ laminar diffusivity, $\mathrm{m}^{2} / \mathrm{s}$

$D_{\mathrm{T}} \quad$ turbulent diffusivity, $\mathrm{m}^{2} / \mathrm{s}$

$d$ diameter of the jet nozzle, $\mathrm{m}$

$F_{r} \quad$ Froude number

$J$ diffusion vector, $\mathrm{m} / \mathrm{s}$

$k \quad$ global sensitivity of the detection system for one particle (signal level)

$N_{\mathrm{P}} \quad$ number of seed particles in the probe volume

$N_{\mathrm{P}, i} \quad$ number of seed particles in sample $\mathrm{i}$

$N_{\mathrm{P}}{ }^{(i)} \quad$ value of $N_{\mathrm{P}}$ in channel $i$

$N_{\mathrm{B}} \quad$ number of Doppler bursts

$\dot{N}_{\mathrm{B}} \quad$ count rate of bursts, $\mathrm{s}^{-1}$

$N_{\mathrm{v}} \quad$ number of validated Doppler bursts

$\dot{N}_{\mathrm{V}} \quad$ count rate of validated bursts, $\mathrm{s}^{-1}$

$N_{\text {id }} \quad$ number of ideal particles

$N_{\text {id }}{ }^{*} \quad$ number of marked ideal particles

$P^{\star}$ probability that an ideal particle be marked by a seed particle

$P(\rho \mathrm{z}) \quad$ probability density function for $\rho \mathrm{z}, \mathrm{m}^{3} / \mathrm{kg}$

$P_{\left(N_{\mathrm{P}}=k\right)} \quad$ probability to have $k$ seed particles in the probe volume
$P_{\left(N_{\mathrm{P}}=k \mid \rho z\right)}$ $r$ $R_{\rho}$ $S_{1}$ $S_{1}^{(1)}$ $s_{1}$ $v_{\mathrm{c}}$ $V$ $V_{\mathrm{x}}$ $V_{\mathrm{r}}$ $V_{\mathrm{P}}$ $V_{\mathrm{P} j}$ $V_{\mathrm{P} i j}$

$V_{i}$ $V_{1, i}$ $x$

$Y_{i}$

Z

$Z_{i}$

Greek
$\rho$
$\rho_{i}$
$\rho^{(1)}$
$\tau_{1}$
$\tau_{\mathrm{B}}$

Averages

$<A>$

$\overline{\bar{A}}$

$A^{\prime \prime}$ probability of having $k$ seed particle conditioned on a given value of $\rho \mathrm{z}$

radial coordinate, $\mathrm{m}$ $=\rho^{(1)} / \rho^{(2)}$, density ratio local signal level with jet seeding reference signal level in pure stream 1 with jet seeding

$=S_{1 /} S_{1}{ }^{(1)}$, normalized signal

volumic capacity of the probe volume, $\mathrm{m}^{3}$ velocity vector, $\mathrm{m} / \mathrm{s}$ axial velocity component, $\mathrm{m} / \mathrm{s}$ radial velocity component, $\mathrm{m} / \mathrm{s}$ particulate velocity vector, $\mathrm{m} / \mathrm{s}$ velocity vector of particle $j, \mathrm{~m} / \mathrm{s}$ velocity vector of the $j$ th particle in sample $i$, $\mathrm{m} / \mathrm{s}$ velocity vector of the marked flow for realization $i, \mathrm{~m} / \mathrm{s}$

velocity vector of the flow such it is marked in realization $i$ by particles issuing only from stream $1, \mathrm{~m} / \mathrm{s}$ axial coordinate, $\mathrm{m}$ local mass fraction of species $i$ mixture fraction:local mass fraction of jet fluid mixture fraction for realization $i$

local density, $\mathrm{kg} / \mathrm{m}^{3}$

local density for realization $i, \mathrm{~kg} / \mathrm{m}^{3}$

density in stream 1 (density of the jet fluid), $\mathrm{kg} / \mathrm{m}^{3}$

time of flight of jet seed particles to reach the probe volume, $s$

duration of a Doppler burst, $s$

ensemble average of $A$

time average of $A=\overline{\bar{A}},(\overline{\bar{A}} \equiv \tilde{A})$ the present notation is only due to printing problems Favre fluctuation, $A^{\prime \prime}=A-\overline{\bar{A}}$

Received: 5 December 2002 / Accepted: 10 June 2003 
(Bilger 1976; Pratt 1976). At first sight, $Z$ appears as a simple local scalar quantity, but actually the definition above is functional, as conditioning on the fluid origin involves implicit Lagrangian aspects linked to the history experienced by the fluid before it reaches the probe volume. The mixture fraction quantifies the global scalar state of the mixture over a local probe volume, but alone it cannot provide information on the mixing that proceeds within the control volume. Locally, the action of mixing implicates different velocities of the fluid elements according to their origin, and joint local measurements of conditional velocities and mixture fractions are required to study the mixing dynamic.

In laminar flows where the fluid is homogeneously composed of a huge number of single molecules, the continuity equation can be applied to the conservation of the mass of molecules originating from the jet stream, and molecular mixing comes out in a diffusion flux

$J=Z(1-Z)\left(V_{1}-V_{2}\right)$

where $V_{1}$ is the local velocity vector of the fluid originating from the jet, $V_{2}$ is that of the fluid originating from the ambient, and the global convection velocity is defined by

$V=Z V_{1}+(1-Z) V_{2}$

Notice that $V$ is defined as a mass weighted composition of the conditional velocities so that local momentum conservation is implicit in the transport equation for the mass of injected fluid.

For uniform density flows, statistical calculations (kinetic theory of gases) show that

$J=-D_{\mathrm{L}} \nabla Z$

where $D_{\mathrm{L}}$ is the laminar diffusivity of the flow.

In turbulent flows, averaging of the conservation equations for the mass of injected fluid leads to additional correlation terms between $\rho, Z$, and velocity components. When using Favre decomposition, these correlation terms reduce to $\overline{\overline{Z^{\prime \prime} V^{\prime \prime}}}$. By formal analogy with the gradient-diffusion in a laminar flow with constant density, the additional Favre correlation term called turbulent transport of the mixture fraction is usually modeled by

$\overline{\overline{Z^{\prime \prime} V^{\prime \prime}}}=-D_{\mathrm{T}} \nabla \overline{\bar{Z}}$

where $D_{\mathrm{T}}$ is a turbulent diffusivity, and where it is expected that Favre averaging will properly account for density fluctuations (Bilger 1979).

Unfortunately, direct experimental assessment of gradient-diffusion modeling in turbulent flows is extremely difficult because simultaneous measurement of $Z$ and $V$ is still a challenge due to the practical incompatibility (seeding conditions) of the two diagnostics (Starner 1983; Dibble et al. 1987).

In the near development field of turbulent mixing flows where sharp gradients are expected, measurement of the scalar and velocity fields must be performed with high spatial, temporal, and dynamic resolutions. The present investigation in turbulent jets with strong density differences between the injected fluid and the ambient has been made in two steps and with two optical techniques: pulsed laser Mie scattering for measurement of the mixture fraction, and laser Doppler velocimetry (LDV). In each technique, the laser light is scattered by seed particles that are added either to the jet fluid or to the ambient air in a slow coflow. The seed particles are assumed to follow the turbulent motion of the fluid. For both techniques, particular attention has been brought to seeding conditions and to the statistical differences that may result from unequal number density of seed particle in the mixing flow, especially for LDV, in which the sampling is not random, as the acquisition of a velocity data is determined by the passage of a particle in the probe volume.

Careful and detailed experiments in turbulent jets (Dibble et al. 1987; Labacci et al. 1988; Stepowski and Sautet 1998) have shown that the ensemble average velocities obtained when only the jet fluid was seeded are higher than those obtained when only the coflow air was seeded (especially for the radial components). This difference in the conditional LDV data was considered as a seeding bias, and correction procedures were proposed by Dibble et al. (1987) to restore the unconditional velocity from the two conditional ones, but no attempt was made to obtain an analytic expression of this difference.

The naive idea that has guided our investigations is that the difference in the mean conditional velocities could be merely the signature of the action of turbulent mixing within the control volume in the early stages of the jet development. The present work analyzes the difference in the velocity statistics conditioned on jet seeding and on coflow seeding in order to derive data on the dynamics of mixing in the near field of turbulent jets with variable density. Complementarily, the structure and the mean field of the mixture fraction have been explored by laser Mie scattering with conditional seeding.

\section{Experimental arrangement}

\section{1}

\section{Turbulent jets and seeding}

The experimental setup schematized in Fig. 1 has been described in previous papers (Sautet and Stepowski 1994, 1995; Stepowski and Sautet 1998). It consists of a vertical pipe ( $d=10 \mathrm{~mm}, L=1 \mathrm{~m}$ ) fed with various mixtures of hydrogen and nitrogen (bulk density $\rho^{(1)}$ ) flowing in a regime of fully developed turbulence. The jet exit velocity $V_{\mathrm{x}}{ }^{(1)}$ is fixed for all experiments at $45 \mathrm{~m} / \mathrm{s}$ with a turbulence level of $4.5 \%$ (in terms of rms of the fluctuating component of the injection velocity versus its mean value). Several jet mixtures were used so as to vary the initial density ratio $\mathrm{R}_{\rho}=\rho^{(1)} / \rho^{(2)}$ relative to the ambient from 0.07 up to 1 , while the Reynolds number varied from 4,100 up to 27,000 .

The jets are discharging into a slow coflow air stream $\left(V_{\mathrm{x}}{ }^{(2)}=4.5 \mathrm{~m} / \mathrm{s}\right)$ which is properly conditioned by a honeycomb section followed by a convergent duct (exit section $=100 \mathrm{~cm}^{2}$ ). The coflow Reynolds number is 27,000 and its turbulence level is $2 \%$.

The jet and the coflow can be separately seeded at large upstream distance with nebulized silicon oil droplets 


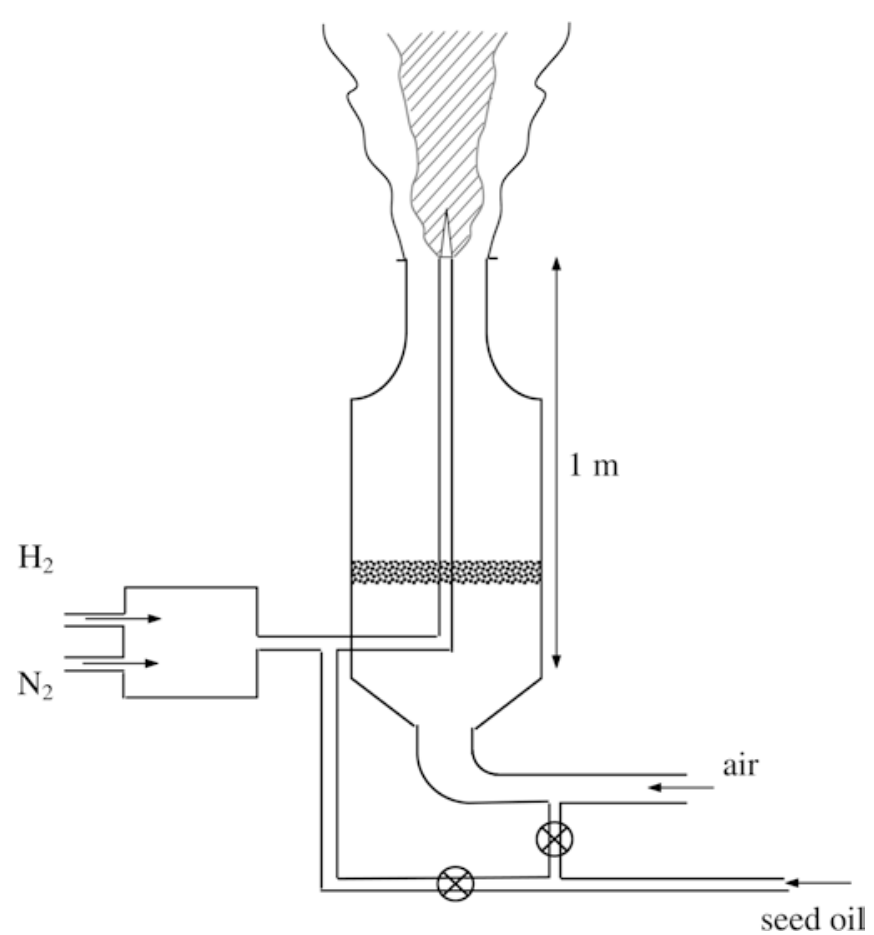

Fig. 1. Experimental setup

(diameter $\approx 1 \mu \mathrm{m}, \rho=1.1 \mathrm{~g} / \mathrm{cm}^{3}, v=500 \mathrm{~mm}^{2} / \mathrm{s}$ ). Based on their Stokes number, these particles are able to track accurately the fluctuations of the fluid motion up to $0.2 \mathrm{MHz}$. This seeding system is used for laser Mie scattering as well as for LDV with the same kind of particle, but the seeding rate is reduced to a very low level for LDV.

\section{2}

\section{Laser Mie scattering}

Radial profiles of the mixture fraction and density at various axial stations in the turbulent jets have been obtained by laser Mie scattering with conditional seeding.

A pulsed YAG laser ( $\lambda=532 \mathrm{~nm}, F=10 \mathrm{~Hz}, \Delta t=8 \mathrm{~ns})$ is horizontally focused at a given station along a diameter of the jet with a beam waist of $0.2 \mathrm{~mm}$. At each laser shot, the laser light scattered by the seed particles is collected at $90^{\circ}$ and focused onto a 1-D gated intensified diode array consisting of 1,024 pixels ( $25 \mu \mathrm{m}$ wide each).

Part of the incident laser beam is split and focused into the potential core of the jet (where $Z=1, \rho=\rho^{(1)}$ ) to induce a reference scattering signal. At each laser firing, the reference signal level is used to correct for drifts in the jet seeding rate and shot to shot fluctuations of the laser energy. For jet seeding operations, it is assumed that the seeding rate does not vary during the short time (about $5 \mathrm{~ms}$ ) taken by the fluid to reach the measurement station. The validity of this hypothesis will be discussed in Sect. 3.1. For coflow seeding operations, no reference beam is required, as the normalization is provided in situ by the signal level at the outward edges of each radial profile where $Z=0$, and $\rho=\rho^{(2)}=\rho_{\text {air }}$.

For these Mie scattering measurements the seeding rate was adjusted to have about $10^{5}$ particle in the probe volume which corresponds to a mass fraction of particles lower than $0.5 \%$. These conditions insure that the particles do not modify the fluid properties (including no noticeable optical extinction) while keeping a low marker shot noise of about $1 \%$.

For each station in the jet development, 700 instantaneous profiles are registered at $10 \mathrm{~Hz}$. The mean profile over these realizations is an ensemble average, because the medium stays frozen during each laser pulse and the repetition rate of the sampling is much lower than the turbulence frequency (random sampling).

\section{3}

\section{Laser Doppler velocimetry}

LDV has been performed with a dual color dual beam system. Axial and radial velocity components are measured using the green line and the blue line of an argonion laser. Directional ambiguity in the radial component is eliminated by frequency shifting $(40 \mathrm{MHz}$ on each beam pair). The four beams are focused using a $480 \mathrm{~mm}$ focal length lens. The spatial resolution is $500 \mu \mathrm{m}$ (radial) by $90 \mu \mathrm{m}$ (axial and azimuthal).

Particular attention was paid to the seeding conditions: alternatively, one of the two channels was seeded (solely the jet stream then solely the coflow) at a low rate with great care to avoid residual particles in the other channel. As detailed in Sect. 4.2, the seeding rate was reduced to have a validation ratio better than $90 \%$ and to make sure that further reduction of the seeding rate did not improve this validation ratio. This caution insured that the probability to have more than one particle in the probe volume was negligible. To obtain the ensemble average velocity data, 1,500 samples were used.

\section{3}

\section{Measurement of the scalar field}

\section{1}

\section{Principle of the measurement}

The laser Mie scattering technique has been intensively used (Becker 1977; Ebrahimi and Kleine 1977; Long et al. 1881; Stepowski and Cabot 1988) to measure the density in turbulent flows. In a flow seeded with identical particles, the local mass fraction $Y_{\mathrm{P}}$ of particles is related to the particulate population $N_{P}$ by

$Y_{\mathrm{P}}=N_{\mathrm{P}} W_{\mathrm{P}} / \rho v_{\mathrm{C}}$

where $W_{\mathrm{P}}$ is the mass of one particle, $v_{\mathrm{C}}$ is the probed volume, and $\rho$ is the local density of the fluid.

As the seed particles are assumed to track accurately the turbulent fluid motion without perturbing it $\left(Y_{\mathrm{p}}<<1\right)$, with the assumption of a marker continuum (marker shot noise $<1 \%$ ), the mass fraction of particle is a conserved property that can be linearly expressed as a function of the mixture fraction

$Y_{\mathrm{P}}(t)=Z(t) Y_{\mathrm{P}}^{(1)}\left(t-\tau_{1}\right)+[1-Z(t)] Y_{\mathrm{P}}^{(2)}\left(t-\tau_{2}\right)$

where $Y_{\mathrm{P}}{ }^{(i)}\left(t-\tau_{i}\right)$ is the mass fraction of particles in pure stream $i$ and $\tau_{i}$ is the time taken by the fluid to reach the measurement station (index 1 refers to the jet stream, index 2 refers to the coflow). 
The local number of particles in the probe volume is then given by

$N_{\mathrm{P}}(t)=N_{\mathrm{P}}^{(1)}\left(t-\tau_{1}\right) \frac{\rho(t) Z(t)}{\rho^{(1)}}+N_{\mathrm{P}}^{(2)}\left(t-\tau_{2}\right) \frac{\rho(t)[1-Z(t)]}{\rho^{(2)}}$

where $N_{\mathrm{P}}{ }^{(i)}\left(t-\tau_{I}\right)$, and $\rho^{(i)}$ are the number of particles at $t-\tau_{i}$ and the fluid density respectively in pure stream $i$. The intensity of the scattered light is proportional to the local number of particles, to the constant scattering cross section of the particles and to the laser irradiance.

When only the jet is seeded $\left(N_{\mathrm{P}}^{(2)}=0\right)$ the local signal level is

$S_{1}(t)=k N_{\mathrm{P}}^{(1)}\left(t-\tau_{1}\right) \frac{\rho(t) Z(t)}{\rho^{(1)}}$

and the reference signal level from the jet exit where $Z=1$ and $\rho=\rho^{(1)}$ is

$S_{1}^{(1)}(t)=k N_{\mathrm{P}}^{(1)}(t)$

The normalized signal is then given by

$s_{1}(t)=\frac{S_{1}(t)}{S_{1}^{(1)}(t)}=\frac{N_{\mathrm{P}}^{(1)}\left(t-\tau_{1}\right)}{N_{\mathrm{P}}^{(1)}(t)} \frac{\rho(t) Z(t)}{\rho^{(1)}}$

Complementarily, when only the coflow is seeded $\left(N_{\mathrm{P}}{ }^{(1)}=0\right)$, the local signal level is

$S_{2}(t)=k N_{\mathrm{P}}^{(2)}\left(t-\tau_{2}\right) \frac{\rho(t)[1-Z(t)]}{\rho^{(2)}}$

and the reference signal level from the outward edge of the radial profile where $Z=0$ and $\rho=\rho^{(2)}$ is

$S_{2}^{(2)}(t)=k N_{\mathrm{P}}^{(2)}\left(t-\tau_{2}\right)$

The normalized signal is then given by

$s_{2}(t)=\frac{S_{2}(t)}{S_{2}^{(2)}(t)}=\frac{\rho(t)[1-Z(t)]}{\rho^{(2)}}$

These two complementary conditional measurements cannot be performed simultaneously. In addition to

Eq. (10) or (13), an other relationship between $\rho$ and $Z$ is needed to derive the instantaneous profiles of density and mixture fraction. At constant temperature and constant pressure the local specific volume is given by

$\frac{1}{\rho}=\frac{Z}{\rho^{(1)}}+\frac{(1-Z)}{\rho^{(2)}}$

As the analysis of the uncertainties (Sautet and Stepowski 1994) shows that coflow seeding is more suitable in the near development field, the instantaneous radial profiles of density and mixture fraction are derived from

$\rho(t)=\left[\rho^{(2)}-\rho^{(1)}\right] s_{2}(t)+\rho^{(1)}$

$Z(t)=1-\frac{\rho^{(2)}}{\rho(t)} s_{2}(t)$
For mean values, in terms of ensemble average, it is better to use the two mean conditional data (Eqs. 10 and 13) to derive the mean density because our results show that $\left\langle s_{1}\right\rangle+\left\langle s_{2}\right\rangle=1$ within $2 \%$ accuracy, which validates (via Eq. 14) the assumption that on average during the short delay $\tau_{1}$ taken by the jet fluid to reach the measurement station, drifts in seeding rate are negligible. Then,

$\langle\rho\rangle=\rho^{(1)}\left\langle s_{1}\right\rangle+\rho^{(2)}\left\langle s_{2}\right\rangle$

and the ensemble Favre average of the mixture fraction is derived from

$\frac{\langle\rho Z\rangle}{\langle\rho\rangle}=1-\frac{\rho^{(2)}}{\langle\rho\rangle}\left\langle s_{2}\right\rangle$

\section{2}

\section{Results and discussion}

Examples of instantaneous and average radial profiles of the mixture fraction at two axial stations $(x / d=0.5$ and 10) are shown in Fig. 2 for a pure hydrogen jet $\left(\rho^{(1)} / \rho^{(2)=} 0.07\right)$, and in Fig. 3 for a pure nitrogen jet. According to Chen and Rodi (1980) and Chassaing (1979), the axisymmetric flow is a pure inertial jet as far as $x / d<0.5\left(\mathrm{~F}_{\mathrm{r}}\right)^{1 / 2}\left[\rho^{(1)} / \rho^{(2)}\right]^{1 / 4}$. The length of the inertial region is $30 d$ for the pure nitrogen jet and $9 d$ for the pure hydrogen jet. In both cases, the radial profiles across the exit section looks like a top hat function. However, in the early stages of the jet development, the instantaneous profiles are discontinuous and contain sharp peaks and valleys with different widths. Since the profiles at the neck of the injector appear as a homogeneous top hat function, the strong inhomogeneities observed in the near field of the mixing flow cannot be attributed to marker shot noise or to spatial resolution

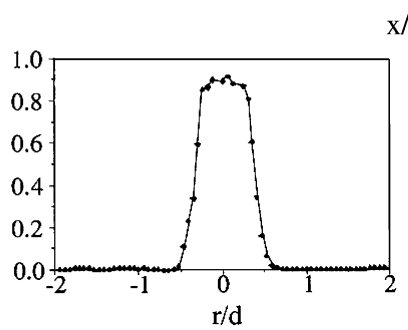

$\mathrm{x} / \mathrm{d}=0.5$
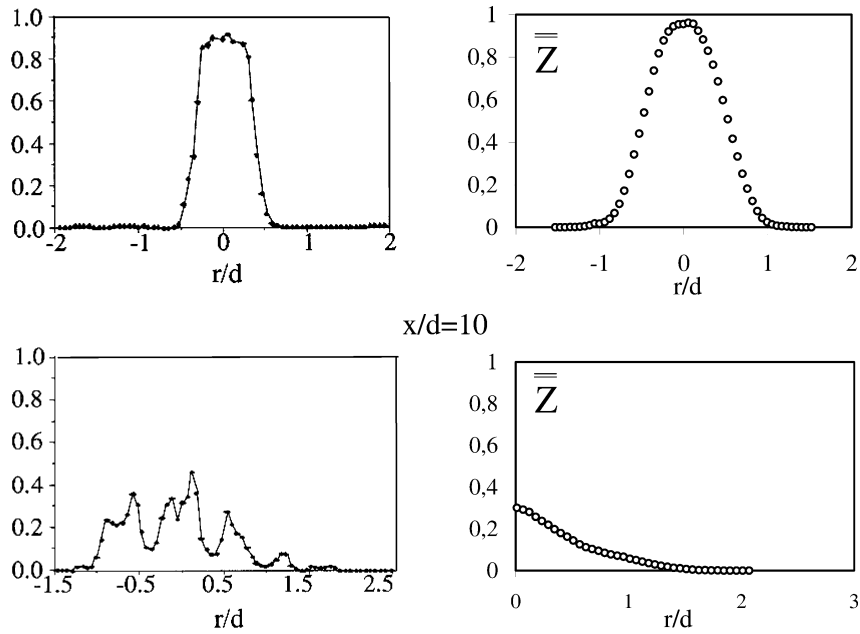

$\mathrm{x} / \mathrm{d}=10$

Fig. 2. Examples of instantaneous (right-hand side) and average (left-hand side) radial profiles of the mixture fraction at $x / d=0.5$ (top) and $x / d=10$ (bottom) for a pure hydrogen jet $\left(R_{\rho}=0.07\right)$ 


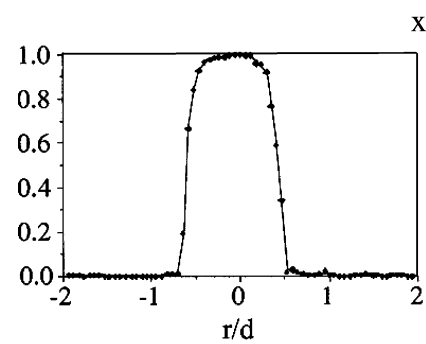

$\mathrm{x} / \mathrm{d}=0.5$

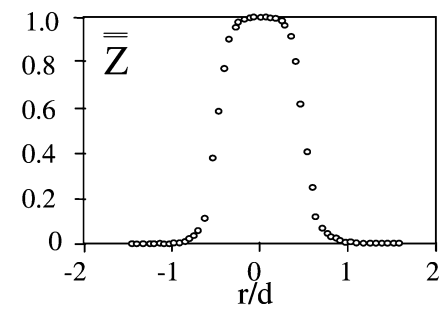

$\mathrm{x} / \mathrm{d}=10$
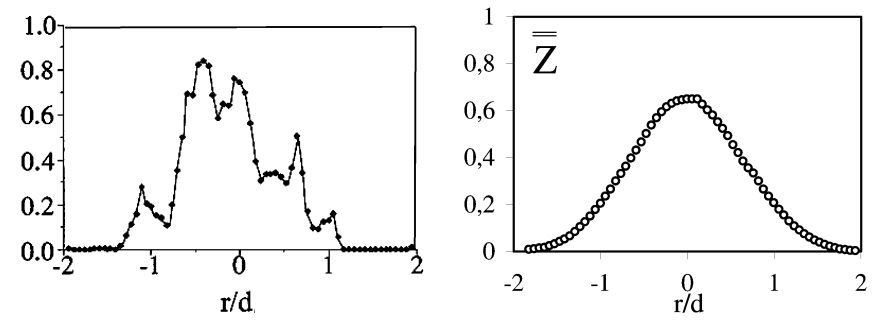

Fig. 3. Examples of instantaneous (right-hand side) and average (left-hand side) radial profiles of the mixture fraction at $x / d=0.5$ (top) and $x / d=10$ (bottom) for a nitrogen jet $\left(R_{\rho}=1\right)$

problems. The separation between the scale of these scalar inhomogeneities and the scale of the mean flow is not large, and this is generally considered (Tennekes and Lumley 1972) as the main limitation of gradient-diffusion modeling in turbulent jets. These instantaneous profiles (Figs. 2a, 3a) show that various mixed fluid compositions can be found in structures with different extents and that unmixed ambient fluid can be sometimes entrained deep in the jet. As also observed and commented by Dahm and Dimotakis (1987), the strong inhomogeneities in the instantaneous profiles-whereas the average profiles are continuous functions as in a laminar flow-point out an essential difference between molecularly mixed fluid at a given composition and an average over mixed an unmixed fluids yielding the same mean composition. The basic difference could be due to the dynamic effects induced by the coherent structures of a turbulent flow when mixing proceeds. A turbulent flow involves the motion and collision of groups of molecules rather than only those of individual molecules as in laminar flows (Struminskiy 1987); this difference is generally admitted-even if sometimes forgotten-although the tenuous nature of this grouping is still a matter of discussions: according to Pope (2000), the consistency between the random nature of turbulent flows and the deterministic nature of classical mechanics embodied in the Navier-Stokes equations is due to the extreme sensitivity of these equations to the slightest variation in initial conditions; beyond a given spatio-temporal domain the flow properties cannot be predicted, and the molecules have to be regrouped over such domains where their macroscopic scalar and vectorial properties are continuously predictable and defined.

Since the instantaneous scalar profiles are irregular in the early stages of the turbulent mixing flow, it can be expected that the associated conditional velocity fields would be also inhomogeneous. Under such conditions, the validity of gradient-diffusion modeling is doubtful since it is based on analogy with molecular diffusion in laminar flows for which homogeneity is essential. In the following, the mean velocities conditioned on the two origins of the marked fluid will be investigated to see whether averaging homogenizes the conditional velocities as it does for the scalar, and whether data on the local mixing dynamics may be derived from differences in the mean conditional velocities.

\section{4}

\section{LDV with conditional seeding}

LDV measures the velocity of single particles as they cross the probe volume. In most cases, identical particles are artificially added to the flow, and the velocity of a particle is assumed to represent the local fluid velocity. The mean local velocity provided by the LDV system is an ensemble average velocity, and usually the residence time of each particle in the probe volume is not accounted for (nor the mass of each probed particle) in the averaging. However, this ensemble average needs to be specified as the frequency of the events (i.e., passage of a particle in the probe volume) is linked to the local concentration of seed particles that have been transported by the flow. For conditional velocity measurements where solely one of the two streams is seeded the frequency of these events will be linked to the local concentration of particles originating from the seeded channel.

\section{1}

\section{General principle of conditional velocimetry}

In this section the principle of conditional velocimetry is analyzed with the assumption of a marker continuum and apart for technical constraint (i.e., without the LDV requirement to have only one particle in the probe volume) when measuring the particulate velocities. The ensemble average velocity over $\mathrm{k}$ particles is

$$
\left\langle V_{\mathrm{P}}\right\rangle=\frac{1}{k} \sum_{j=1}^{j=k} V_{\mathrm{P} j}
$$

Actually, the measurement of $\left\langle V_{\mathrm{P}}>\right.$ must be performed by an ensemble of $q$ realizations in a given probe volume. If $N_{\mathrm{P} i}$ particles are present in the probe volume for realization $i$, a mean velocity $V_{i}$ is attributed to the marked flow for realization $i$ according to

$V_{i}=\frac{1}{N_{\mathrm{P} i}} \sum_{j=1}^{j=N_{\mathrm{P} i}} V_{\mathrm{P} i j}$

where $V_{\mathrm{P} i j}$ is the velocity of the $j$ th particle in sample $i$. The ensemble average particulate velocity over the $q$ realizations (i.e., over the $k$ particles) is then given by

$\left\langle V_{\mathrm{P}}\right\rangle=\frac{\sum_{i=1}^{i=q} N_{\mathrm{P} i} V_{i}}{\sum_{i=1}^{i=q} N_{\mathrm{P} i}}$

which can be expressed as 


$$
\left\langle V_{\mathrm{P}}\right\rangle=\frac{\left\langle N_{\mathrm{P} i} V_{i}\right\rangle}{\left\langle N_{\mathrm{P} i}\right\rangle}
$$

where $V_{i}$ is the velocity of the marked flow for realization $i$, and $N_{\mathrm{P} i}$ is given by Eq. (7).

For jet seeding,

$$
N_{\mathrm{P} i}=N_{\mathrm{P}}\left(t_{i}\right)=N_{\mathrm{P}}^{(1)}\left(t_{i}-\tau_{1 i}\right) \frac{\rho\left(t_{i}\right) Z\left(t_{i}\right)}{\rho^{(1)}}
$$

For coflow seeding,

$N_{\mathrm{P} i}=N_{\mathrm{P}}\left(t_{i}\right)=N_{\mathrm{P}}^{(2)}\left(t-\tau_{2 i}\right) \frac{\rho\left(t_{i}\right)\left[1-Z\left(t_{i}\right)\right]}{\rho^{(2)}}$

As the local flow properties $(\rho, Z, V)$ are not a priori correlated with the populations $N_{\mathrm{P}}{ }^{(1)}$ or $N_{\mathrm{P}}{ }^{(2)}$ of seed particle in the initial streams, the ensemble average particulate velocities that are measured with jet seeding (subscript 1) and coflow seeding (subscript 2 ) are given respectively by

$$
\begin{aligned}
\left\langle V_{\mathrm{P}}\right\rangle_{1} & =\frac{\left\langle\rho_{i} Z_{i} V_{1, i}\right\rangle}{\left\langle\rho_{i} Z_{i}\right\rangle} \\
\left\langle V_{\mathrm{P}}\right\rangle_{2} & =\frac{\left\langle\rho_{i}\left(1-Z_{i}\right) V_{2, i}\right\rangle}{\left\langle\rho_{i}\left(1-Z_{i}\right)\right\rangle}
\end{aligned}
$$

where for each sampling $i, V_{1, i}$ is the velocity of the flow such as it is marked by particles issuing only from stream 1 (jet), whereas $V_{2, i}$ is the velocity of the flow such as it is marked by particles issuing from stream 2 (coflow).

Dynamic homogeneity of the marked flow has not been assumed to yield these expressions in which the sampled velocities of the marked flow are still specified by the origin of the marker. However, notice that, even if dynamic homogeneity were assumed (i.e., $V_{1, i=} V_{2, i}$ ), the two conditional mean velocities would still have different expressions. The difference in the velocities conditioned on the marker origin cannot be only considered as a technical bias since expressions (25) and (26) have been obtained with the assumptions of both ideal marker and ideal velocimeter.

This basic result (Eq. 25 for instance) merely yields that for jet seeding each sampled velocity of the marked flow has been weighted by the corresponding density of jet fluid, as well as the mean local number of markers is proportional to the mean local density, $\langle\rho \mathrm{Z}\rangle$, of jet fluid. In shorthand, this operation can be called ensemble conditional Favre averaging.

However, the LDV technique is not able to provide such a mean velocity of the marked flow for the events where several particles are present in the control volume (LDV is basically incompatible with the assumption of a marker continuum). It applies only when a single particle crosses the probe volume, and it provides the velocity of that single particle. Events $i$ with $N_{\mathrm{P}, i}>1$ are not validated and not accounted for in the ensemble averaging.

For jet seeding, as the particles follow the turbulent motion of the fluid, if $\left\langle N_{\mathrm{P}}^{(1)}\right\rangle>1$, the local condition $N_{\mathrm{P}}=1$ will be preferentially achieved for events when the local concentration of jet fluid is lower than its mean local value, leading to an overestimate of these events in the local ensemble average velocity. In contrasr for low rate of jet seeding, $\left\langle N_{\mathrm{P}}^{(1)}\right\rangle<<1$, one can ask whether the condition $N_{\mathrm{P}}=1$ would over-weight the contribution of events with local high value of jet fluid concentration in the ensemble average of LDV data (actually it will not).

Careful analysis of the statistical distribution of the number of seed particles in the probe volume is needed to quantify these biases, since both the triggering of the velocimeter and the post-validation of the LDV data are determined by this number.

\section{2}

\section{Marker statistics in LDV with conditional seeding}

The seed particles are assumed to follow the turbulent motion of the fluid without disturbing it. However, for LDV the number $N_{\mathrm{P}}$ of seed particles in the control volume corresponding to a validated acquisition of velocity is zero or one in total contradiction with the assumption of a marker continuum. The discrete nature of the marking then comes into evidence through a random fluctuation or "shot noise" (Becker et al. 1967; Shaughnessy and Morton 1977).

Assume first that the fluid density and motion could be continuously portrayed-apart from molecular agitation-by a high number $N_{\text {id }}$ of ideal particles. The actual number of seed particles $N_{\mathrm{P}}$ can be considered as the number $N_{\mathrm{id}}{ }^{*}$ of ideal particles that are marked. $N_{\mathrm{id}}{ }^{*}$ is then binomially distributed with $P^{*}$ being the probability that an ideal particle be marked by a seed one. Then $<N_{\mathrm{P}}>=<N_{\mathrm{id}}{ }^{*}>=<N_{\mathrm{id}}>P^{*}$ with $P^{*}<<1$. Under these conditions, the statistical distribution of $N_{\mathrm{P}}$ is locally given by the Poisson law

$P_{\left(N_{\mathrm{P}}=k\right)}=\frac{\left\langle N_{\mathrm{P}}\right\rangle^{k}}{k !} \mathrm{e}^{-\left\langle N_{\mathrm{P}}\right\rangle}$

The following analysis will consider the case of jet seeding (for coflow seeding $N_{\mathrm{P}}^{(1)} / \rho^{(1)}$ is replaced by $N_{\mathrm{P}}^{(2)} / \rho^{(2)}$ and $Z$ is replaced by $\left.[1-Z]\right)$. The next step is to express the local ensemble average population $\left\langle N_{\mathrm{P}}\right\rangle$ as a function of the initial mean population in the jet fluid $\left\langle N_{\mathrm{P}}^{(1)}\right\rangle$, and of the local mean properties of the turbulent mixing flow. As the principle of ergodicity applies only for random processes, relation (7) for a marker continuum will be used with $N_{\mathrm{P}}^{(2)}=0$ (jet seeding) and only for a given local value of $\rho \mathrm{Z}$. Then when the probed volume contains a high number of particles (marker continuum), relation (7) conditioned on a given local value of $\rho \mathrm{Z}$ gives

$$
\frac{\rho Z}{\rho^{(1)}}=\frac{N_{\mathrm{P}}\left(t_{i}\right)}{N_{\mathrm{P}}^{(1)}\left(t_{i}-\tau_{i}\right)}=\frac{\sum_{i=1}^{q} N_{\mathrm{P}}\left(t_{i}\right)}{\sum_{i=1}^{q} N_{\mathrm{P}}^{(1)}\left(t_{i}-\tau_{i}\right)}=\frac{\sum_{i=1}^{q} N_{\mathrm{P} i}}{\sum_{i=1}^{q} N_{\mathrm{P} i}^{(1)}}
$$

If the volume over which local and initial particulate populations are probed is divided into $m$ equal subvolumes such that the instantaneous populations $N_{\mathrm{P} k i}$ and $N_{\mathrm{P} k i}^{(1)}$ in a sub-volume $n$ can be small numbers (possibly one or zero for high value of $m$ ), then 


$$
\begin{aligned}
\frac{\rho Z}{\rho^{(1)}}= & \frac{\sum_{i=1}^{q} \sum_{n=1}^{m} N_{\mathrm{P} i n}}{\sum_{i=1}^{q} \sum_{n=1}^{m} N_{\mathrm{P} i n}^{(1)}}=\frac{\sum_{n=1}^{m} \sum_{i=1}^{q} N_{\mathrm{P} i n}}{\sum_{n=1}^{m} \sum_{i=1}^{q} N_{\mathrm{P} i n}^{(1)}} \\
= & \frac{q \sum_{n=1}^{m}\left\langle N_{\mathrm{P} i}\right\rangle_{n}}{q \sum_{n=1}^{m}\left\langle N_{\mathrm{P} i}^{(1)}\right\rangle_{n}} \stackrel{\text { (ergodicity) }}{=} \frac{\left\langle N_{\mathrm{P} i}\right\rangle_{n}}{\left\langle N_{\mathrm{P} i}^{(1)}\right\rangle_{n}}
\end{aligned}
$$

where the number of particle in the probe volume $n$ for an event $i$ can be as small as wanted provided that ensemble averages $\left\langle N_{\mathrm{P} i}\right\rangle_{n}$ and $\left\langle N_{\mathrm{P} i}^{(1)}\right\rangle$ are performed over a high number of samples (high value of $q$ ). For jet seeding, the probability that $k$ particles are present in the probe volume is then conditioned on the local value of $\rho \mathrm{Z}$ according to

$P_{\left(N_{\mathrm{P}}=k \mid \rho Z\right)}=\frac{\left(\frac{\left\langle N_{\mathrm{p} i}^{(1)}\right\rangle \rho Z}{\rho^{(1)}}\right)^{k}}{k !} \mathrm{e}^{-\frac{\left\langle N_{\mathrm{P}}^{(1)}\right\rangle \rho Z}{\rho^{(1)}}}$

Applying the total probability theorem (Bayes) over all possible values of $k$ and $\rho Z$, the ensemble average velocity of particles originated from the jet stream is found to be the same as in Eq. (25)

$$
\left\langle V_{\mathrm{P}}\right\rangle_{1}=\frac{\int_{\rho z} \sum_{k=1}^{\infty} k P_{\left(N_{\mathrm{P}}=k \mid \rho z\right)} V_{1}(\rho z) P(\rho z) \mathrm{d}(\rho z)}{\int \sum_{\rho z} \sum_{k=1}^{\infty} k P_{\left(N_{\mathrm{P}}=k \mid \rho z\right)} P(\rho z) \mathrm{d}(\rho z)}=\frac{\left\langle\rho_{i} Z_{i} V_{1, i}\right\rangle}{\left\langle\rho_{i} Z_{i}\right\rangle}
$$

where $V_{1}$ is the velocity of the flow such as it is marked by particles issuing only from stream 1 , and $P(\rho \mathrm{z})$ is the local probability density function of $\rho \mathrm{z}$.

Now when the velocity measurements are performed by LDV, the summation over $k$ is restricted to $k=1$ by the validation procedure, and then

$$
\begin{aligned}
\left\langle V_{\mathrm{LDV}}\right\rangle_{1} & =\frac{\int P_{\left(N_{\mathrm{P}}=1 \mid \rho z\right)} V_{1}(\rho z) P(\rho z) \mathrm{d}(\rho z)}{\int P_{\left(N_{\mathrm{P}}=1 \mid \rho z\right)} P(\rho z) \mathrm{d}(\rho z)} \\
& =\frac{\left\langle\rho_{i} Z_{i} V_{1, i} \mathrm{e}^{-\left\langle N_{\mathrm{P}}^{(1)}\right\rangle \rho_{i} Z_{i} / \rho^{(1)}}\right\rangle}{\left\langle\rho_{i} Z_{i} \mathrm{e}^{-\left\langle N_{\mathrm{P}}^{(1)}\right\rangle \rho_{i} Z_{i} / \rho^{(1)}}\right\rangle}
\end{aligned}
$$

Thus for jet seeding, LDV may under-estimate the contribution of events with high values of $\rho \mathrm{Z}$ with respect to the expected ensemble conditional Favre average (Eq. 25) in as much as $N_{\mathrm{P}}{ }^{(1)}$ is high. In the worst case of high seeding rate $\left(N_{\mathrm{P}}{ }^{(1)}>>1\right)$, LDV with jet seeding would paradoxically give the mean velocity associated with intermittent engulfment of unmixed ambient air in the probe volume.

In contrast, for low seeding conditions $\left(N_{\mathrm{P}}{ }^{(1)}<<1\right)$, the bias becomes negligible and LDV still provides the ideal ensemble conditional Favre average velocity. However, in this regime of low seeding rate of one channel, great care must be taken to avoid any residual seed particle in the other input channel.
A criterion for this regime of low seeding to be reached is available through the validation rate displayed by the LDV system.

\subsection{1}

\section{Validation ratio and seeding rate}

The counter gives the cumulative brute number of bursts $N_{\mathrm{B}}$ (for which $N_{\mathrm{P}} \geq 1$ ), the cumulative number of validated Doppler bursts $N_{\mathrm{V}}$ (for which $N_{\mathrm{P}}=1$ ), and the validation ratio $\alpha=N_{\mathrm{V}} / N_{\mathrm{B}}$. The probability to have $N_{\mathrm{P}} \geq 1$ is given by the proportion of time over which $N_{\mathrm{P}} \geq 1$ :

$P_{\left(N_{\mathrm{P}} \geq 1\right)}=\frac{\left\langle N_{\mathrm{B}}\right\rangle\left\langle\tau_{\mathrm{B}}\right\rangle}{T}=\dot{N}_{\mathrm{B}}\left\langle\tau_{\mathrm{B}}\right\rangle=\dot{N}_{\mathrm{B}}\left\langle\frac{h}{V}\right\rangle$

where $\dot{N}_{\mathrm{B}}$ is the brute count rate (in s ${ }^{-1}$ ), and $h$ is the spatial resolution in the direction of the flow velocity $V$. Then

$P_{\left(N_{\mathrm{P}} \geq 1\right)}=1-P_{\left(N_{\mathrm{P}}=0\right)}=1-\mathrm{e}^{-\left\langle N_{\mathrm{P}}\right\rangle}=\left\langle\frac{h}{V}\right\rangle \dot{N}_{\mathrm{B}}$

and

$P_{\left(N_{\mathrm{P}}=1\right)}=\left\langle N_{\mathrm{P}}\right\rangle \mathrm{e}^{-\left\langle N_{\mathrm{P}}\right\rangle}=\left\langle\frac{h}{V}\right\rangle \dot{N}_{\mathrm{V}}$

where $\dot{N}_{\mathrm{V}}$ is the count rate of validated Doppler bursts (in $\mathrm{s}^{-1}$ ). In the potential core of the jet where $\rho \mathrm{Z}=\rho^{(1)}$, the validation ratio is

$\alpha^{(1)}=\frac{\dot{N}_{\mathrm{V}}^{(1)}}{\dot{N}_{\mathrm{B}}^{(1)}}=\left\langle N_{\mathrm{P}}^{(1)}\right\rangle \frac{\mathrm{e}^{-\left\langle N_{\mathrm{p}}^{(1)}\right\rangle}}{1-\mathrm{e}^{-\left\langle N_{\mathrm{P}}^{(1)}\right\rangle}}$

Under our low seeding conditions, the LDV counter gave $\alpha^{(1)}=0.9$ such that $\left\langle N_{\mathrm{P}}^{(1)}\right\rangle \approx 0.2$. Actually this criterion is too severe because some Doppler bursts with $N_{\mathrm{P}}=1$ may be still rejected for technical problems. Then

$\langle h / V\rangle \dot{N}_{\mathrm{V}}=P_{\left(N_{\mathrm{P}}=1\right)} \times P^{\prime}$

where $P^{\prime}$ is the probability that a burst from a single particle be validated to provide a velocity data. Thus actually

$\alpha=\frac{\left\langle N_{\mathrm{P}}\right\rangle \mathrm{e}^{-\left\langle N_{\mathrm{P}}\right\rangle}}{1-\mathrm{e}^{-\left\langle N_{\mathrm{P}}\right\rangle}} \times P^{\prime}$

A more realistic estimate of $\left\langle N_{\mathrm{P}}\right\rangle$ is obtained by a differential study of $\alpha$ when $\dot{N}_{\mathrm{B}}$ varies. As

$\frac{\partial \ln \dot{N}_{\mathrm{B}}}{\partial\left\langle N_{\mathrm{P}}\right\rangle}=\frac{\mathrm{e}^{-\left\langle N_{\mathrm{P}}\right\rangle}}{1-\mathrm{e}^{-\left\langle N_{\mathrm{P}}\right\rangle}}$

calculations show that

$\frac{\partial \ln \alpha}{\partial \ln \dot{N}_{\mathrm{B}}}=\frac{\mathrm{e}^{\left\langle N_{\mathrm{P}}\right\rangle}\left(1-\left\langle N_{\mathrm{P}}\right\rangle\right)-1}{\left\langle N_{\mathrm{P}}\right\rangle} \approx-\frac{\left\langle N_{\mathrm{P}}\right\rangle}{2}$ if $\left\langle N_{\mathrm{P}}\right\rangle<<1$

In the potential core of the jet, we have obtained $\frac{\Delta \ln \alpha^{(1)}}{\Delta \ln \dot{N}_{\mathrm{B}}^{(1)}} \approx-3 \%$, then $\left\langle N_{\mathrm{P}}^{(1)}\right\rangle \approx 0.06$ insuring that $\mathrm{e}^{-\left\langle N_{\mathrm{P}}^{(1)}\right\rangle} \geq 0.94$. Under such seeding conditions, LDV provides the ideal mean conditional velocity given by Eq. (25). 


\section{3}

\section{Results and interpretations}

Figure 4 shows examples of radial profiles of the ensemble average conditional LDV data for the axial component at stations $x=1.5 d, x=5 d, x=15 d$ in the developments of nitrogen and hydrogen jets. The solid lines indicate data collected when seed particles were added only to the jet fluid (subscript 1), the dotted lines indicate data collected when seed particles were added only to the ambient air (subscript 2). On the centerline when LDV data can be collected either by one or the other seeding procedure, the difference between the conditional axial velocities is negligible $\left(<V_{x, \mathrm{P} \mathrm{z}} \approx\left\langle V_{x, \mathrm{P}}\right\rangle_{2}\right)$ as was also observed by Dibble et al. (1987). At a given station $x$, with increasing radius $\left\langle V_{x, \mathrm{P}}\right\rangle_{1}-\left\langle V_{x, \mathrm{P}}\right\rangle_{2}$ increases significantly in the hydrogen jet and reaches a maximum at radius $r_{M}(x)$, whereas it keeps a weak value in the nitrogen jet. With increasing station above the nozzle in the hydrogen jet the difference between the conditional velocities decays: At station $x=1.5 d$, the maximum difference $\Delta V_{\mathrm{x}}$ is $12 \mathrm{~m} / \mathrm{s}$ (at $\left.r_{\mathrm{M}} \approx 0.25 d\right)$, at station $5 d \Delta V_{\mathrm{x}}=3.2 \mathrm{~m} / \mathrm{s}\left(\right.$ at $\left.r_{\mathrm{M}} \approx 0.75 d\right)$, at station $15 d \Delta V_{\mathrm{x}}=1.4 \mathrm{~m} / \mathrm{s}\left(\right.$ at $\left.r_{\mathrm{M}} \approx 1.7 d\right)$. Notice that different velocity scales have been used to plot the profiles in

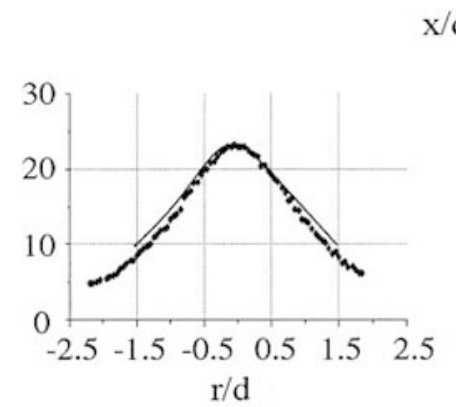

$$
\mathrm{x} / \mathrm{d}=15
$$
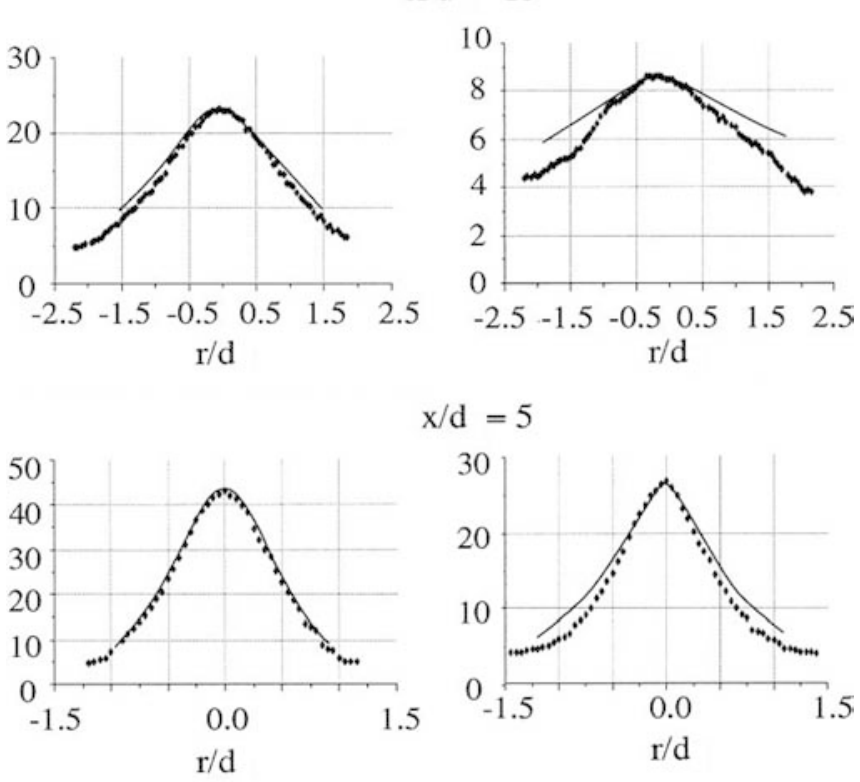

$$
\mathrm{x} / \mathrm{d}=5
$$

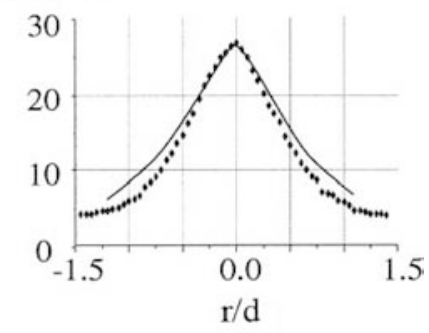

$$
\mathrm{x} / \mathrm{d}=1.5
$$

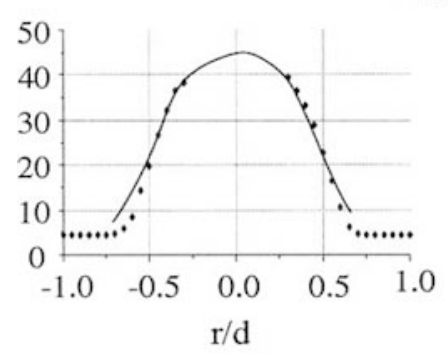

Nitrogen jet

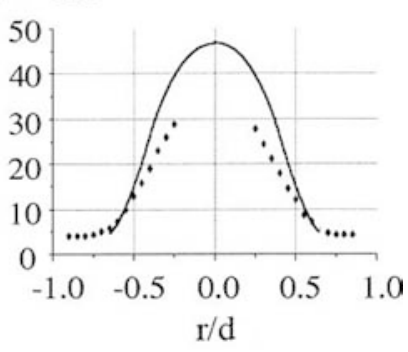

Hydrogen jet
Fig. 4. Radial profiles of conditioned LDV data (in $\mathrm{m} / \mathrm{s}$ ) for the axial component at three stations in the early stages of the turbulent mixing flow: solid lines, jet seeding; dotted lines, coflow seeding the nitrogen jet and in the hydrogen jet because the centerline decay of the axial velocity is faster in the hydrogen jet than in the nitrogen jet. Based on conservation of mean mass and momentum, the self-similar decay of the centerline velocity is a hyperbolic function of

$\left(x-x_{0}\right) / d \sqrt{\rho^{(1)} / \rho^{(2)}}$

(Chen and Rodi 1980), where the abscissa of the fictive origin is higher for the nitrogen jet $\left(x_{0} \approx-1 d\right)$ than for the hydrogen jet $\left(x_{0} \approx-5 d\right)$ (Sautet and Stepowski 1995). Therefore, regarding axial velocities the rate of turbulent mixing is higher for the low density hydrogen jet $\left(\sqrt{\rho^{(2)} / \rho^{(1)}}=3.84\right)$ especially in the near development field where mixing proceeds earlier since $x_{0}$ is smaller for the hydrogen jet. This scaling difference in the self-similar decay for the hydrogen and nitrogen jets is consistent with the observed resemblance between the profile at $5 d$ in the hydrogen jet and the profile at $15 d$ in the nitrogen jet, although the difference in conditional velocities is still lower in the nitrogen jet.

Figure 5 shows the profiles of conditional radial velocities in the same jets. Noticeable differences between the conditional velocities are observed in both jets although the difference is still lower (factor 0.5) in the nitrogen jet. The absolute magnitude of the difference in
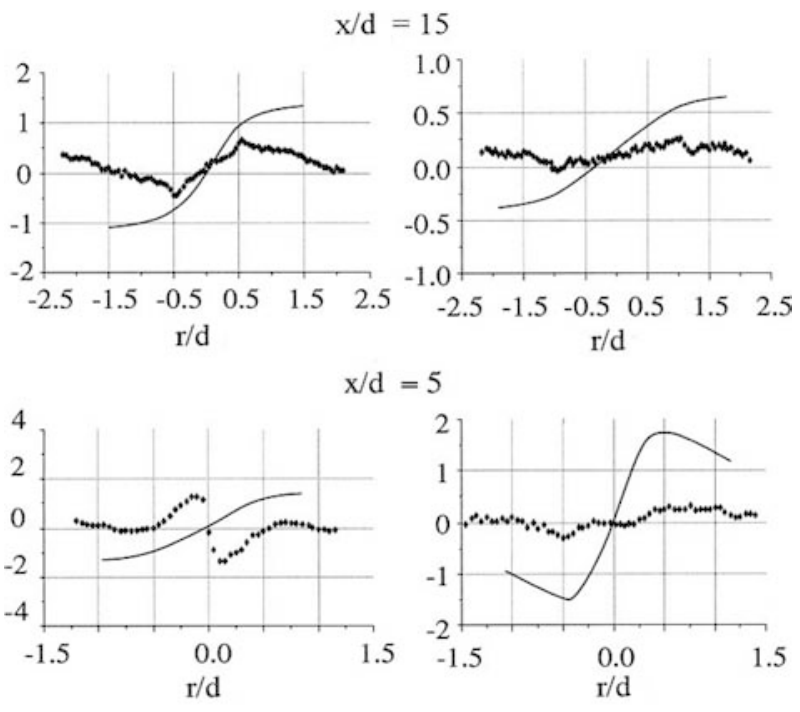

$\mathrm{x} / \mathrm{d}=1.5$

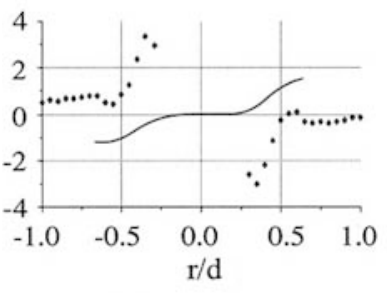

Nitrogen jet

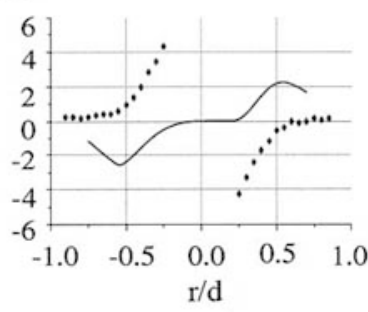

Hydrogen jet
Fig. 5. Radial profiles of conditioned LDV data (in $\mathrm{m} / \mathrm{s}$ ) for the radial component at three stations in the early stages of the turbulent mixing flow: solid lines, jet seeding; dotted lines, coflow seeding 
conditional radial velocities is rather lower than for the axial component in the hydrogen jet, but observation of the relative radial profiles reveal striking features: In the earlier stages of the turbulent mixing layer for both jets $\left\langle V_{\mathrm{r}, \mathrm{P}}\right\rangle_{1}$ is centrifugal whereas $\left\langle V_{\mathrm{r}, \mathrm{P}}\right\rangle_{2}$ is centripetal! At downstream stations, both conditional velocities are centrifugal but $\left\langle V_{\mathrm{r}, \mathrm{P}}\right\rangle_{1}$ is still higher than $\left\langle V_{\mathrm{r}, \mathrm{P}}\right\rangle_{2}$. The difference in conditional velocities decays with increasing distances from the nozzle. In the hydrogen jet at station $x=1.5 d$ the maximum velocity difference $\Delta V_{\mathrm{r}}$ is $5 \mathrm{~m} / \mathrm{s}$ (at $r_{\mathrm{M}} \approx 0.25 d$ ), at station $5 d \Delta V_{\mathrm{r}}=1.4 \mathrm{~m} / \mathrm{s}$ (at $\left.r_{\mathrm{M}} \approx 0.5 d\right)$, at station $15 d \Delta V_{\mathrm{r}}=0.5 \mathrm{~m} / \mathrm{s}\left(\right.$ at $\left.r_{\mathrm{M}} \approx 1.5 d\right)$. Thus, for the hydrogen jet, the relative axial decay of the maximum difference between the conditional radial velocities is practically the same as for the axial components, and the radius of maximum difference increases with increasing axial stations as the jet develops.

These observations corroborate our presumption (conjecture) that the local difference between the velocity data conditioned on the origin of the marker (Eqs. 25, 26) contains quantitative information on the dynamic of mixing within the control volume.

If the turbulent mixing flow is locally assumed to be statistically stationary and homogeneous with respect to the origin of the fluid, ensemble average reduces to time average (ergodicity) and $V_{1, i}=V_{2, i}=V_{i}$ so that the local difference between the conditioned LDV data reduces to

$$
\left\langle V_{\mathrm{P}}\right\rangle_{1}-\left\langle V_{\mathrm{P}}\right\rangle_{2}=\frac{\overline{\rho Z V}}{\overline{\rho Z}}-\frac{\overline{\rho(1-Z) V}}{\overline{\rho(1-Z)}}=\frac{\overline{\overline{Z^{\prime \prime} V^{\prime \prime}}}}{\overline{\bar{Z}}(1-\overline{\bar{Z})}}
$$

Now, with the assumption of homogeneity the turbulent transport of $Z$ can be approximated (Williams 1985) by gradient-diffusion (Eq. 4) leading to

$$
\left\langle V_{\mathrm{P}}\right\rangle_{1}-\left\langle V_{\mathrm{P}}\right\rangle_{2} \approx-D_{\mathrm{T}} \frac{\nabla \overline{\bar{Z}}(1-\overline{\bar{Z}})}{\overline{\bar{Z}}\left(1-{ }^{2}\right.}
$$

The difference between the radial components of the ensemble average conditional LDV data have been plotted as a function of $-\frac{\partial \bar{Z}}{\partial r} / \overline{\bar{Z}}(1-\overline{\bar{Z}})$ using the profiles of ensemble Favre average scalar, $\langle\rho Z\rangle /\langle\rho\rangle \approx \bar{Z}$, provided by conditional laser Mie scattering measurements in the same flow. Close to the nozzle where the radial profile of $\bar{Z}$ looks like a top hat function, the accuracy on the ratio $\frac{\partial \overline{\bar{Z}}}{\partial r} / \overline{\bar{Z}}(1-\overline{\bar{Z}})$ is low excepted in two narrow regions of strong gradient. In contrast, for downstream stations $(x / d>20)$ both the difference in conditional velocities and the radial gradient of $\bar{Z}$ have low values that do not allow for relevant validation of relation (41). The optimum compromise is obtained for intermediate stations between $5 d$ and $15 d$. The data shown in Fig. 6 at station $x / d=10$ for two different densities of injected fluid $\left(R_{\rho}=0.53\right.$ and 0.25$)$ gather reasonably well along a common straight line for both jets. The slope of this linear evolution $\left(\approx 3 \times 10^{-3} \mathrm{~m}^{2} / \mathrm{s}\right)$ is in good agreement with the order of magnitude of turbulent diffusivity (Hinze 1975) commonly used in fluid dynamics $\left(D_{\mathrm{T}} \approx 10^{-2} U^{(1)} d\right)$. The accuracy on these data is limited (about 15\%), mainly because the experiments were not initially intended for measurement of a turbulent diffusivity

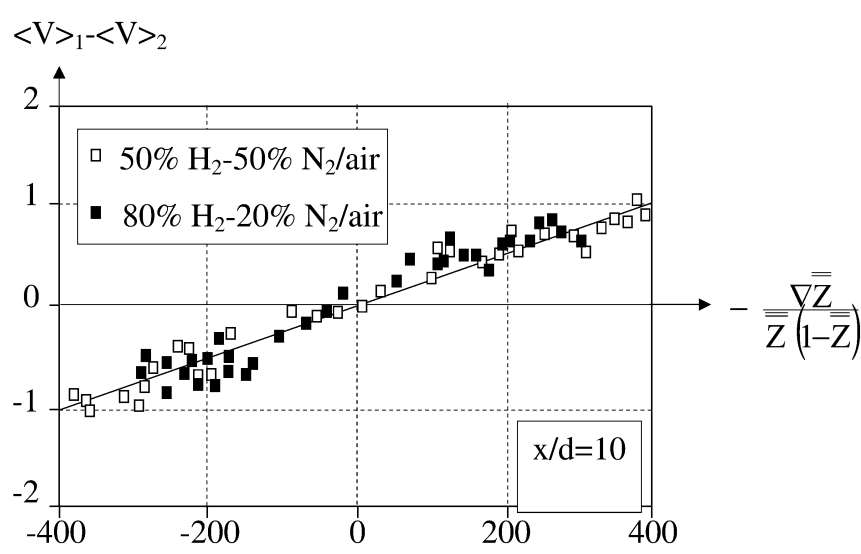

Fig. 6. Diffusivity plot of $\langle V\rangle_{1}-\langle V\rangle_{2}$ as a function of $(-\nabla \overline{\bar{Z}}) / \overline{\bar{Z}}(1-\overline{\bar{Z}})$ at $x / d=10$ for $R_{\rho}=0.25$ (black squares) and $R_{\rho}=0.53$ (white squares), in $\mathrm{m} / \mathrm{s}$ vs $\mathrm{m}^{-1}$ units

(data files for $\mathrm{H}_{2}$ and $\mathrm{N}_{2}$ jet fluids were not exploitable). However, notice that no scale correction or reduced coordinate accounting for density variation was needed to collapse the data along a common straight line, whereas such a variable length scale (effective nozzle diameter) was required to obtain a common self-similar velocity decay for these two jets (Sautet and Stepowski 1998).

These results show that Favre averaging is efficient to account for density fluctuations in the modeling of turbulent transport of scalar by the gradient diffusion approach with the concept of turbulent diffusivity. It is also shown that the local difference in the ensemble average LDV data conditioned on the two different origins of the marked fluid is a quantitative measure of the turbulent mixing in terms of $\overline{Z^{\prime \prime} V^{\prime \prime}}$ (notice that with the assumptions of local dynamic homogeneity used in this interpretation turbulent mixing was reduced to the turbulent transport of the mixture fraction given by the correlation above).

However, the good agreement with gradient-diffusion model in the early stages of the jet development is surprising because in this region the instantaneous profiles of $Z$ showed strong discontinuities (see Fig. 2a, 3a) that are not compatible with the assumptions of homogeneity used in the interpretation. This assumption have been used twice (in Eqs. 40 and 4 to yield Eq. 41) only because our inference was driven in two steps passing by the classical correlation term $\overline{\overline{Z^{\prime \prime}} V^{\prime \prime}}$. Actually, this intermediate is not required and somewhat ill-advised. Instead, for being consistent with the observed inhomogeneity and intermittency of the scalar structures, analysis of the conditional LDV data strongly suggests that

$$
\frac{\langle\rho Z\rangle}{\langle\rho\rangle}\left[1-\frac{\langle\rho Z\rangle}{\langle\rho\rangle}\right]\left[\frac{\left\langle\rho Z V_{1}\right\rangle}{\langle\rho Z\rangle}-\frac{\left\langle\rho(1-Z) V_{2}\right\rangle}{\langle\rho(1-Z)\rangle}\right]=-D_{\mathrm{T}} \nabla \frac{\langle\rho Z\rangle}{\langle\rho\rangle}
$$

(where the third factor on the left-hand side is given by $\left.\left.\left\langle\mathrm{V}_{\mathrm{P}}\right\rangle_{1}-\mathrm{V}_{\mathrm{P}}\right\rangle_{2}\right)$ is a general relationship for turbulent mixing by direct analogy with molecular mixing (Eqs. 1, 3) in a laminar flow where

$Z \times[1-Z] \times\left[V_{1}-V_{2}\right]=-D_{\mathrm{L}} \nabla Z$ 
Relationship (42) would be the consequence of an homomorphism between the molecular properties in a laminar mixing flow and their counterparts in a turbulent flow accurately marked by discrete particles (phonons) with ensemble conditional Favre averaging as operator for that mathematical mapping.

The generality of this relationship lies in three features:

1. Ensemble averaging accounts for any event including intermittent ones such as they can be sometimes observed in the instantaneous structures. (But notice that macroscopic properties in the continuum of a laminar flow are also ensemble averages over the properties of a huge number of molecules in a local control volume.)

2. As the velocity of the marked fluid is specified by the origin of the marker, local mixing dynamic within the control volume is also statistically accounted for and turbulent mixing is not restrictively considered as the turbulent transport of the scalar state of the mixture.

3. Favre averaging-and conditional Favre averaging when the involved property is conditioned on the origin-accounts properly for density fluctuations of the marked fluid. Favre averaging is often considered only as a technique to simplify the form of the averaged conservation equations under the pretence that density fluctuations correlations still remain hidden in the reduced formulation. Actually, mass-weighted averaging was introduced by Favre (1969) essentially to account for the inertial nature of the interaction processes involved in fluid dynamics and especially to use momentum $\rho \mathrm{V}$ as a fundamental variable as in all other branches of physics (whereas velocity alone is not really a physical property). Simplification of the equations was only a bonus.

In the limit of local statistical stationarity and dynamic homogeneity of the turbulent mixing as can be found downstream in the far field, Eq. (42) reduces to the classical gradient-diffusion modeling of the scalar-velocity correlation (Eq. 4) as turbulent mixing is approximated by the turbulent transport of the mixture fraction. It would be helpful to use PIV with conditional seeding (in PIV, the mean conditioned velocity data are no longer weighted by $\rho Z$ or $\rho(1-Z)$, as the sampling is randomly determined by the laser firing) in a turbulent air jet discharging in ambient air to see whether approximation $\left\langle V_{1}\right\rangle \approx\left\langle V_{2}\right\rangle \approx\langle V\rangle$ (i.e., Eq. $42 \equiv$ Eq. 40 ) can still be made in the early stages of the turbulent mixing layer or if only relation (42) is valid. As relation (42) is only based on a homomorphism between the macroscopic molecular properties in a laminar mixing flow and their counterpart (in ensemble average) in a turbulent mixing flow such as it is marked by discrete particles, its validity should not be restricted to shear flows. In perspective work, it would be interesting to perform a basic experiment on turbulent mixing in the near development field of a turbulent jet discharging in a coflow with the same mean velocity.

Arguments supporting the general validity of relationship (42) will be developed in a forthcoming paper. These arguments are implicitly involved when addressing the problem of measurement and definition of the mean unconditional velocity in a turbulent mixing flow.

\section{5}

\section{Summary and concluding remarks}

Analysis of marker statistics in LDV with conditional seeding of one of the two input channels has provided expressions for the two complementary ensemble average conditional velocities in a turbulent mixing flow. With the assumption of stationarity and dynamic homogeneity, the local difference in the mean conditional velocities is a quantitative measure of the turbulent transport of the mixture fraction as given by the classical Favre correlation $\overline{Z^{\prime \prime} V^{\prime \prime}}$. Experimental data in the near development field of turbulent jets with strong density variations confirm the validity of gradient-diffusion modeling for this correlation and the efficiency of Favre averaging to account for the inertial nature of the interaction processes with momentum-rather than velocity-as relevant variable.

Beyond this experimental assessment, to be consistent with the observed inhomogeneity of the scalar structure in the near field, the results suggest the validity of a more general gradient-diffusion relation involving ensemble conditional Favre average velocities. In this form, the flow velocity (as marked by discrete particles) is still specified by the origin of the sampled fluid so as to account also for local mixing dynamic within the control volume.

In this study, according to the fundamental and functional definition of the mixture fraction, the flow properties have been specified by the origin of the fluid elements rather than by their innate nature as given species.

In laminar flows, owing to the randomness of molecular agitation, the origin of a molecule is not macroscopically accessible, and the only way to know it is to use an innate feature (chemical species) as a mark bearing witness to this origin in Eqs. (1) and (2). Then assuming that collisional properties (molecular weight, cross sections etc.) of the molecules are independent of that innate mark, it can be shown (using the total probability theorem over all possible origins) as a consequence that gradient-diffusion relation (3), where the fluid elements (single molecules) are specified by their origin, is valid as well with specification based on any innate feature, $i$, of the molecules in the general form $Y_{i}\left(V_{i}-\mathrm{V}\right)=-D \nabla Y_{i}$. Now when the collisional properties of the molecules depend on their innate nature, this relation (and Eq. 3) are no longer exact. Among the supplementary terms involved in the multicomponent diffusion-equation (Williams 1985), speciesspecific diffusion coefficients $D_{i j}$ are introduced to statistically account for binary collisions between different species. However, although differential diffusion may induce very important phenomena, it must not mask the fundamental role of specification based on the origin.

It is shown in the present work, in turbulent flows where the fluid elements are groups of molecules rather than individual molecules, the origin of these elements, such as they are accurately tracked in their turbulent motion by discrete seed particles, is macroscopically accessible on average by using conditional seeding. It is worth noticing a useful feature of LDV which requires 
having only one particle in the probe volume: Whereas the origin of a given group of molecules (or fluid lump) could not be certified, the origin of the particle that marks a given group of molecules can be certified in a binary way, allowing for simple application of the total probability theorem. In statistical physics (Vauclair 1993), such ideal discrete particles that are used to concretize the collective effect of an ensemble of real elements (although they are different from these real elements) are called phonons.

Finally, LDV with conditional seeding is a relevant observer of turbulent mixing which considers the single particles that mark the motion and density of the turbulent flow with the same point of view (single point statistics) as the molecules are considered in the kinetic theory of gases.

\section{References}

Becker HA (1977) Mixing, concentration fluctuations, and marker nephelometry. In: Launder BE (ed) Studies in convection, vol 1. Academic Press, London, pp 45-139

Becker HA, Hottel HC, Williams GC (1967) On the light-scatter technique for the study of turbulence and mixing. J Fluid Mech 30:259-284

Bilger RW (1976) Turbulent jet diffusion flames. Prog Energy Combust Sci 1:87-109

Bilger RW (1979) Turbulent jet diffusion flames. In:Chigier NA (ed) Energy and combustion science, Stud Ed 1. Pergamon Press, Oxford, pp 109-131

Chassaing P (1980) Melange turbulent de gaz inertes dans un jet tube libre. Thèse de Doctorat, Toulouse, France

Chen CJ, Rodi W (1980) Vertical turbulent buoyant jets. A review of experimental data, Pergamon Press, New York

Dahm WJ, Dimotakis PE (1987) Measurement of entrainment and mixing in turbulent jets. AIAA J 25:1216-1223

Dibble RW, Hartmann V, Schefer RW, Kollmann W (1987) Conditional sampling of velocity and scalars in turbulent flames using simultaneous LDV-Raman scattering. Exp Fluids 5:103-113

Ebrahimi I, Kleine R (1977) The nozzle fluid concentration fluctuation field in round turbulent free jets and jet diffusion flames. Proc Combust Inst 16:1711-1723
Favre A (1969) Statistical equations of turbulent gases. In Lavrentiev MA (ed) Problems of hydrodynamics and continuum mechanics. SIAM, Philadelphia, pp 231-266

Hinze JO (1975) Turbulence. McGraw-Hill, New York

Labacci K, Trinité M, Stepowski D, Cabot G (1988) Conditional LDV in a turbulent diffusion flame. In: Durao DFG (ed) Proceedings of the Fourth International Symposium on Applications of Laser Anemometry to Fluid Mechanics, Session 9. Instituto Superior Tecnico, Lisbon, Portugal, p 5

Long MB, Chu BT, Chang RK (1881) Instantaneous two-dimentional gas concentration measurements by light scattering. AIAA J 19:1151-1157

Pope S (2000) Turbulent flows. Cambridge University Press, Cambridge, UK

Pratt DT (1976) Mixing and chemical reaction in continuous combustion. Prog Energy Combust Sci 1:73-86

Sautet JC, Stepowski D (1994) Single shot laser Mie scattering measurements of the scalar profiles in the near field of turbulent jets with variable densities. Exp Fluids 16:353-367

Sautet JC, Stepowski D (1995) Dynamic behaviour of variable-density, turbulent jets in their near development fields. Phys Fluids 7:2796-2806

Sautet JC, Stepowski D (1998) Evolution of the effective nozzle diameter in the buoyancy free development of turbulent jets with variable density. Exp Fluids 25:280-282

Shaughnessy EJ, Morton JB (1977) Laser light-scattering measurements of particle concentration in a turbulent jet. J Fluid Mech $80: 129-148$

Starner SH (1983) Joint measurements of radial velocity and scalars in a turbulent diffusion flame. Combust Sci Technol 30:145-169

Stepowski D, Cabot G (1988) Laser Mie scattering measurement of mean mixture fraction density and temperature by conditional seeding in a turbulent diffusion flame. Proc Combust Inst 22:619625

Stepowski D, Sautet JC (1998) Near fields of jets with variable density. Recent Res Dev Phys Fluids 1:25-44

Struminskiy VV (1987) Kinetic theory of turbulent flow. Fluid Mech Sov Res 16:17-42

Tennekes H, Lumley JL (1972) A first course in turbulence. The MIT Press, Cambridge, Mass.

Vauclair S (1993) Eléments de physique statistique. Inter Edition, Paris

Williams FA (1985) Combustion theory. Benjamin Cummings, Menlo Park, Calif. 